\title{
1 FINGER REPRESENTATIONS IN PRIMARY SOMATOSENSORY CORTEX ARE \\ 2 MODULATED DURING VIBROTACTILE WORKING MEMORY
}

AUTHORS

Finn Rabe ${ }^{1}$, Sanne Kikkert ${ }^{1,2^{*}}$, Nicole Wenderoth ${ }^{1 *}$

\section{AFFILIATIONS}

${ }^{1}$ Neural Control of Movement Laboratory, Department of Health Sciences and Technology, ETH Zürich, Zürich, Switzerland.

${ }^{2}$ Spinal Cord Injury Center, Balgrist University Hospital, University of Zürich, Zürich, 11 Switzerland.

\section{ABSTRACT}

It is well-established that vibrotactile stimulations elicit Blood-oxygen-level-dependent (BOLD) responses in somatotopically organized brain regions. Whether these somatotopic maps are modulated by working memory (WM) is still unknown. In our WM experiment, participants had to compare frequencies that were separated by a delay period. Vibrotactile stimuli were sequentially applied to either their right index or little finger. Using functional MRI, we investigated whether vibrotactile WM modulated neural activity in primary somatosensory (S1), an area that is known to contain individual finger representations. Our mass-univariate results revealed the well-described network of brain regions involved in WM. Interestingly, our mass-univariate results did not demonstrate $\mathrm{S} 1$ to be part of this network. However, when we parametrically modulated the time-binned regressors in our GLM we found that the delay activity in S1 and secondary somatosensory cortex (S2) was reflected in a U-shaped manner. Using multi-voxel pattern analysis (MVPA), an analysis technique that is 
bioRxiv preprint doi: https://doi.org/10.1101/2021.10.29.466459; this version posted November 1, 2021. The copyright holder for this preprint (which was not certified by peer review) is the author/funder, who has granted bioRxiv a license to display the preprint in perpetuity. It is made available under aCC-BY-ND 4.0 International license.

1 more sensitive to subtle activity differences, we found finger-specific patterns of activation in 2 the $\mathrm{S} 1$ hand area during the WM delay period. These results indicate that processes 3 underlying WM modulate finger-specific representations during our discrimination task. 


\section{INTRODUCTION}

2 Topographic representations such as the somatotopic map in the somatosensory cortex 3 have been shown to be ubiquitous, to a certain extent, in the cerebral cortex of mammals.

4 They consist of orderly representations of receptor surfaces and are present in most of the neocortex (Kaas, 1993, 1997; PENFIELD \& BOLDREY, 1937; Silver \& Kastner, 2009). These somatotopic maps are incredibly specific to the point where the sensation of each finger can be assigned to its own contralateral cortical region, so called finger representations (Besle et al., 2013; Cunningham et al., 2013; Martuzzi et al., 2014; Sanchez Panchuelo et al., 2018; Sanders et al., 2019).

Importantly, the selective activation of these finger representations is not exclusively modulated by tactile stimulation or movement, but also through other mechanisms like (i) through attempted movements, without producing overt motor output (i.e., without any tactile or proprioceptive feedback) (Ariani et al., 2021; Guan et al., 2021; Kikkert et al., 2021) (ii) observed touch (Kuehn et al., 2018), (iii) or directing attention to only one of multiple fingers receiving tactile stimulation (Puckett et al., 2017).

Another mechanism that may modulate $\mathrm{S} 1$ activity is working memory (WM). In the absence of sensory stimuli, decisions are made with information stored in WM (Christophel et al., 2017). Support for S1 involvement in tactile WM stems from neurophysiological research in non-human primates. Single-unit activity was recorded from the S1 hand area while subjects had to match an object with a specific surface to previous presented surface stimulus (Y. D. Zhou \& Fuster, 1996; Y.-D. Zhou \& Fuster, 2000). The authors observed cells that were not only involved in perception of tactile stimuli, but also sustained their firing during the WM delay period. This suggests that primary sensory cortices could serve as a memory buffer for stimulus information (D’Esposito \& Postle, 2015), an idea that has been conceptualized in 'sensory recruitment' models of WM (Katus et al., 2015; Pasternak \& Greenlee, 2005). According to this model, WM is maintained in the same brain regions that are also involved in sensory stimuli encoding. However, human neuroimaging studies on vibrotactile WM do not suggest a selective modulation of activity in S1 during the delay period of information storage (Christophel et al., 2017; Preuschhof et al., 2006). These studies generally employed a mass-univariate approach, which is often not sensitive enough to reveal (i) temporal changes in delay period activity and (ii) specific features of WM content. Concerning the former, information retention in WM is not always reflected in constant delay activity, especially when a temporal structure of the delay period can be anticipated (Rose et al., 2016). Furthermore, it has been shown that persistent delay activity might not be crucial to retain WM content (Stokes, 2015). One approach to more selectively identify brain regions 
1 associated with inconsistent delay activity could be through using model predictors that are

2 graded according to the anticipated delay activity (Braver et al., 1997).

In addition, WM studies employing a mass-univariate approach might often not be sensitive enough to patterns of WM content (Weaverdyck et al., 2020). Instead, multi-voxel pattern analysis (MVPA) has the ability to detect stimulus information in spatially distributed patterns of activation in a region of interest (ROI). In our case, somatotopic maps can be investigated with high sensitivity through combining functional MRI (fMRI) and MVPA. Here, above chance level classification accuracies indicate that brain activation patterns representing single fingers in $\mathrm{S} 1$ are highly specific, in accordance with the idea of narrow tuning curves in the S1 neuronal population (Detorakis \& Rougier, 2014). Lower classification accuracies, by contrast, indicate that $\mathrm{S} 1$ activity is less finger specific suggesting that tuning curves are broader. Importantly, MVPA accuracies can reveal representational information even if the average Blood-oxygen-level-dependent (BOLD) response does not surpass a certain statistical threshold in univariate analyses.

Neuroimaging studies utilizing MVPA found that features like vibratory frequencies were solely represented during the delay period in associative brain regions, i.e., posterior parietal and frontal regions (Schmidt et al., 2017; Wu et al., 2018). Interestingly, features of texture stimuli could be decoded from S1 delay period activity (Schmidt \& Blankenburg, 2018). Similar to the aforementioned observations in non-human primates, decoded textures were located in the contralateral S1 hand area, an area which has shown to possess finegrained finger representations (Besle et al., 2013; Cunningham et al., 2013; Martuzzi et al., 2014; Sanchez Panchuelo et al., 2018; Sanders et al., 2019). This suggests that neural activity in S1 during the delay period may not only reflect some stimulus feature, but also a resides within somatotopically organized cortical areas.

Here we used $\mathrm{FMRI}$ combined with MVPA to explore whether finger representations in contralateral S1 are selectively modulated through WM maintenance, i.e., in the absence of any tactile input. Participants were asked to perform a vibrotactile frequency discrimination task on the index or little finger while we collected $\mathrm{fMRI}$ data. We hypothesized that if WM selectively activates $\mathrm{S} 1$, then we will observe finger selective activation patterns during the WM delay period. 
1 Thirty young healthy volunteers (19 females; mean age $=24.48, \mathrm{SEM}=0.44$ ) participated in

2 our study. Our sample size was comparable to those in previous reports on $\mathrm{fMRI}$ decoding

3 of WM content using discrimination tasks (Ester et al., 2009; Schmidt et al., 2017). All

4 participants were neurologically intact and reported to be right-handed. All of them gave

5 written informed consent and the study protocol was approved by the local ethics committee

6 (BASEC-Nr. 2018-01078). Three participants had to be excluded due to excessive head

7 motion based on our criterion (see 'Preprocessing of fMRI data' section for more detail).

\section{TACTILE STIMULI}

Vibrotactile stimuli (duration $=2 \mathrm{~s}$, sampling rate $=1 \mathrm{kHz}$ ) were applied to the right index or right little finger using a MR-compatible piezoelectric device (PTS-T1, Dancer Design, UK). We selected these fingers as they have the largest inter-finger somatotopic distance (Besle et al., 2013; Ejaz et al., 2015; Kolasinski et al., 2016; Sanders et al., 2019), allowing us to robustly detect the modulation of somatotopic representations by the WM task. The one bin piezoelectric wafers were mounted to the fingertips using custom 3D-printed retainers that were fixed with a Velcro strap. Participants were asked to report any tingling sensation in case the retainer was mounted too tightly. The stimulation consisted of mechanical sinusoids that were transmitted from the testing computer to the piezoelectric device using a $C$ Series Voltage Output Module (National Instruments) and the in-house NI-DAQmx driver.

To ensure similar task difficulty across runs of the main experiment (Harris et al., 2006), we determined the sensory detection threshold (SDT) for both fingers prior to starting the main experiment. SDT was defined as the stimulation intensity at which the participants detected the stimulus $50 \%$ of the time. We stimulated each finger only once per trial at base frequency $(20 \mathrm{~Hz})$ and participants were asked to press a button upon detection of a stimulation. To reliably estimate SDT, we applied a conventional Bayesian-based Quest procedure (QuestHandler in PsychoPy). After each detected or undetected stimulus the algorithm searched for the most probable psychometric function via maximum likelihood estimation over the course of 25 trials starting with a stimulation amplitude of 0.1 Volts (Watson \& Pelli, 1983). The Weibull psychometric function was calculated using the following formula: 
2 where $x$ is the stimulus intensity in Volts and $T$ is the estimated sensory detection threshold.

3 This procedure was performed prior to the first run. If the percentage of correctly 4 discriminated memory trials in a run was below $60 \%$ or above $90 \%$, then we redetermined 5 the SDT using a shortened version of the Quest procedure. In such a case we started the 6 Quest procedure with the previously determined stimulation intensity to reduce the number 7 of iterations (new iterations $=7$ trials). This procedure was applied to keep task difficulty at 8 comparable levels throughout the experiment.

We analyzed changes in behavioral performance occurred across runs, potentially due to perceptual learning, cooling of the fingertips, or fatiguing effects (Fig. 2B). A repeated measures two-way ANOVA (2 fingers $\times 4$ runs) did not show a significant difference in behavioral performance across runs $\left(F(3,2496)=2.0, p=.11, \eta^{2}<0.01\right)$, no significant difference across fingers $\left(F(1,2496)=.48, p=.49, \eta^{2}<0.01\right)$, and no significant interaction effect $\left(F(3,2496)=.47, p=.47, \eta^{2}<0.01\right)$.

\section{MAIN EXPERIMENTAL TASK}

The main experimental task was generated using PsychoPy (Peirce et al., 2019). The experimental task consisted of memory and no memory trials. During a memory trial, participants performed a two-alternative forced choice (2AFC) discrimination task. Two vibrotactile stimuli were consecutively applied to the same finger (i.e., the index or the little finger), separated by a jittered 6-8s delay. We targeted cutaneous mechanoreceptors that respond to stimulations in the flutter range (Mountcastle et al., 1967). One of two stimuli vibrated at $20 \mathrm{~Hz}$ (2s duration at SDT intensity) while the vibration frequency of the other stimulus varied between 22, 24 or $26 \mathrm{~Hz}$ (same duration and intensity). Participants had to indicate by means of a button press whether the first or the second stimulation was higher in frequency (half of the participants) or whether the first or the second stimulation was lower in frequency (the other half of the participants), following previously published procedures (Pleger et al., 2006, 2008, 2009). Responses were recorded via index and middle finger button presses of the other (left) hand using a MR-compatible fiber optic device. We randomized the order how the response options ( $f 1$ and $\mathrm{f} 2$ ) appeared on the screen on a trial-by-trail basis to prevent somatotopy-dependent anticipatory motor activity. After a 3s response period participants received visual feedback (1s) indicating whether their response was correct (highlighted by green color) or incorrect (red; see Fig 1). Participants were instructed to focus their gaze on the fixation cross in the middle of the screen during the complete trial. 
1 Vibrotactile stimuli trials targeted either the index or the little finger and which finger would 2 be stimulated per trial was counterbalanced across each run.
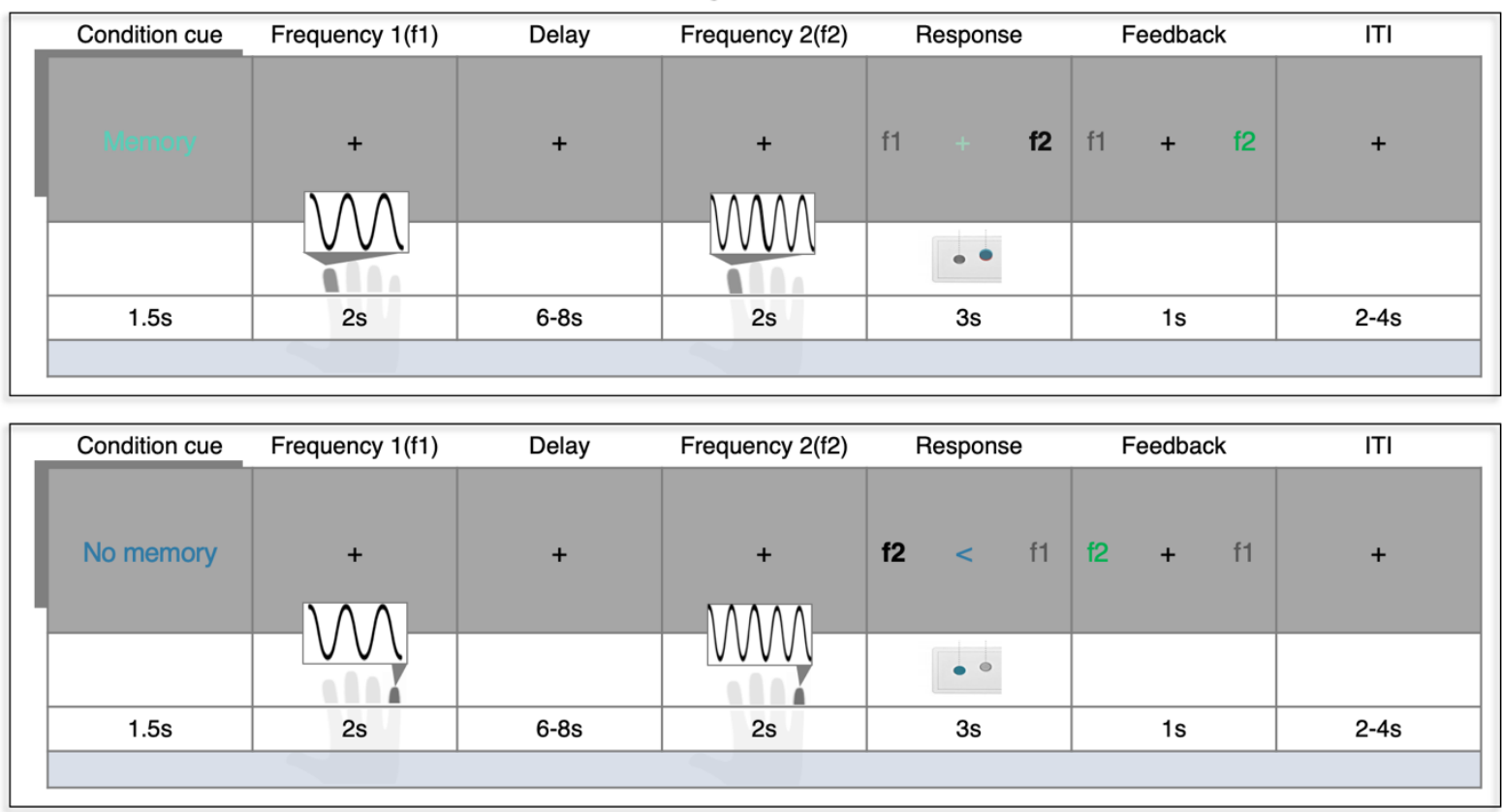

Fig 1. Vibrotactile frequency discrimination task. During memory trials (top) two vibrotactile stimuli that differed in frequency were consecutively applied to the same finger (in this example the index finger). Both stimulations were separated by a jittered delay period, during which participants had to keep the first stimulus (f1) in memory in order to compare it to the subsequent one (f2). During the 3s response period participants had to indicate by means of a left hand button press which of the two stimulation frequencies ( $\mathrm{f} 1$ and $\mathrm{f} 2$ ) was higher. The mapping between the discrimination response and which button to press was randomized across trials. Subjects received feedback whether their response was correct (green) or incorrect (red). The target finger (index or little finger) were intermixed within a run and the inter trial interval (ITI) was jittered between 2-4s. During no memory trials (bottom), vibrotactile stimulations and visual information remained the same. However, participants were instructed not to focus on the stimulation and also not to compare the vibrotactile frequencies. They simply had to press the button indicated by the arrow in the middle of the screen.

To disentangle WM processes from general responses to the stimuli, we also included no memory trials. During a no memory trial participants received the exact same vibratory stimulations as in during memory trials, but they were instructed not to focus on the stimuli or on their vibration frequencies. During the response period subjects were informed by a visual cue (pointing arrow) which button to press. To ensure participants did not switch cognitive strategies, the indicated response was always contrary to the response that would be expected when correctly discriminating both frequencies. Memory and no memory trials conditions were separated in mini blocks of 4 trials. Participants were informed whether they had to perform the memory or no memory task by means of a visual cue $(1.5 \mathrm{~s})$ at the 
1 beginning of each trial. Prior to the experiment, participants were familiarized with the 2 memory and no memory tasks by completing 12 trials.

The order of stimulus sites (stimulated finger) was counterbalanced both within and across mini blocks. Stimulation frequencies were counterbalanced across the experiment.

5 Each stimulus frequency was presented equally often in both memory and no memory 6 condition. Jittered timings for Inter-stimulus-interval (ISI, 6-8s) and Inter-trial-interval (ITI, 27 4s) were randomly drawn from a uniform distribution. All participants completed 4 runs 8 consisting of 48 trials each. Each run consisted of 6 memory and 6 no memory mini blocks 9 in a counterbalanced order.

\section{BEHAVIORAL ANALYSIS}

11 We defined the discrimination accuracy per participant as the percentage of correctly 12 discriminated trials separately for each condition. We expected that greater frequency 13 differences would facilitate discrimination between both tactile vibrations while the stimulus site should have no effect. We therefore investigated whether behavioral performances differed across frequency differences and across fingers using a two-way repeatedmeasures ANOVA.

\section{MRI DATA ACQUISITION}

Functional as well as structural MRI images were acquired on a Philips Ingenia 3 Tesla MRI (Best, The Netherlands) using a 32-element head coil. fMRI data was collected using an echoplanar-imaging (EPI) sequence acquiring 36 transversal slices centred at the bicommissural line and with whole brain coverage, though excluding most of cerebellum (repetition time (TR): $2 \mathrm{~s}$, echo time (TE): $30 \mathrm{~ms}$, spatial resolution: $3 \mathrm{~mm}^{3}, \mathrm{FOV}=222 \times 222 \mathrm{~mm}^{2}, 85^{\circ}$ flip angle, slice orientation: transversal, SENSE factor (AP): 2, 472 functional volumes per run). Anatomical images were acquired during SDT estimation using a MPRAGE T1-weighted sequence $\left(T R=7.7 \mathrm{~ms}, \mathrm{TE}=3.6 \mathrm{~ms}, \mathrm{FOV}=240 \times 240 \mathrm{~mm}^{2}\right.$, flip angle: $8^{\circ}$, resolution: $1 \mathrm{~mm}^{3}$, number of slices: 160 , slice thickness: $2.2 \mathrm{~mm}$, slice orientation: sagittal).

\section{PREPROCESSING OF FMRI DATA}

30 Conventional pre-processing steps for $\mathrm{fMRI}$ data were applied to each individual run in native 31 three-dimensional space, using FSL's Expert Analysis Tool FEAT (v6.00; fsl.fmrib.ox.ac.uk/fsl/fslwiki). The following steps were included: Motion correction using MCFLIRT (Jenkinson, 2002), brain extraction using automated brain extraction tool BET 
1 (Smith, 2002), high-pass filtering $(100 \mathrm{~Hz})$, slice-time correction, and spatial smoothing using

2 a 3mm FWHM (full width at half maximum) Gaussian kernel using FEAT. Functional data was

3 aligned to structural images initially using FLIRT (Jenkinson \& Smith, 2001), and optimised

4 using boundary based registration (Greve \& Fischl, 2009). BOLD EPI data was assessed for

5 excessive motion using motion parameter estimates from MCFLIRT. If the functional data

6 from a participant showed greater than $1.5 \mathrm{~mm}$ (half the voxel size) of absolute mean

7 displacement, this participant was excluded from all further analysis.

To reduce physiological noise artifacts, these CSF and white matter were used to extract scan-wise time series which were then added to the model as nuisance regressors in addition to the standard motion parameters.

11 Structural images were transformed to Montreal Neurological Institute (MNI) standard space using nonlinear registration (FNIRT), and the resulting warp fields were applied to the functional statistical images.

We used each individual participant T1-weighted image to create a cortical surface reconstruction by means of Freesurfer (Fischl et al., 1999). We identified regions of interest area parcellation provided by Freesurfer (Fischl, 2012). More specifically, an S1hand ROI was defined by combining Brodmann areas (BA) 1, 2, 3a, and 3b. We then converted this S1ROI to volumetric space. Any holes were filled and non-zero voxels were mean dilated. Next, the axial slices spanning 2cm medial/lateral to the hand area (Yousry et al., 1997) were identified on the $2 \mathrm{~mm}$ MNI standard brain (min-max MNI z-coordinates=40-62). This mask was nonlinearly transformed to each participant's native structural space. Finally, we used this mask to restrict the S1ROI and extracted an S1hand area ROI. Similar ROI definition has been previously used (Diedrichsen et al., 2013; Ejaz et al., 2015; Wiestler \& Diedrichsen, 2013). The S1hand area ROI was used to both extract time-binned estimates as well as to decode information about the stimulus site during the delay period.

First-level parameter estimates were computed per run using a voxel-based general linear model (GLM) based on the gamma hemodynamic response function. Time series statistical analysis was carried out using FILM (FMRIB's Improved Linear Model) with local autocorrelation correction. 
To find neural correlates of WM we contrasted beta estimates from the delay period during memory trials to those in no memory trials. We then used a fixed effects higher-level analysis to average activity across runs for each individual participant. Finally, to make inferences on the population level, we computed a mixed effects analysis (Flame 1). From this we obtained statistical group maps (Z-statistic images) for each contrast of interest, e.g. contrasting memory delay activity to no memory delay activity. Z-statistic images were thresholded using clusters determined by $Z>3.1$ and $p<.05$ family-wise-error-corrected (FWE) cluster significance.

To further explore whether finger specific activity levels were maintained in a somatotopic fashion, we first computed somatotopic ROls by contrasting finger-specific activity during the first stimulation. We did this by contrasting activity associated with right index stimulations to right little finger stimulations, which elicited a finger specific map in the lateral part of S1while the reverse contrast revealed more medially located activity (see supplementary materials, Fig. 1). These S1activity maps were in line with previous findings on finger somatotopy.

We then compared delay period activity levels between trials where either the index or the little finger was stimulated within each finger ROI. Again, we computed a fixed-effects analysis as mentioned before. We extracted the percent signal change (PSC) separately for each participant using the Featquery tool in FSL.

Information retention in WM is not always reflected by constant delay activity, especially when the duration of the delay period can be somewhat anticipated (Rose et al., 2016). Indeed, persistent delay activity may not be crucial to retain WM content (Stokes, 2015). WM delay activity has been shown to decrease until shortly before memory retrieval when the remembered stimulus information is reactivated as suggested by an increase of neural oscillations in the theta band (Rose et al., 2016). We therefore hypothesized that the BOLD activity level would vary in a U-shaped fashion over the delay period. To test this hypothesis, we conducted a parametric modulation analysis. Our parametric modulation regressor was modelled to predict activity in three consecutive time-bins of the delay period delay period in a U-shaped manner (Fig. 3A). The length of each time-bin equalled one TR 30 (i.e., 2s). Since we jittered the delay period between 6 and $8 \mathrm{~s}$, we only modelled the first 31 three time-bins of the delay period (2-6s). The remaining time of the delay was modelled as 32 a separate regressor of no interest. Z-statistic images were thresholded using clusters 33 determined by $Z>3.1$ and a familywise error-corrected cluster significance threshold of $p<$ 0.05 was applied to the suprathreshold clusters. 
To further visualize the results of the parametric modulation, we extracted activity estimates per time-bin. To do so, we modelled each time-bin of the delay period separately in a voxel-based general linear model (GLM) based on the gamma hemodynamic response function. The remaining time of the delay was modelled as a separate regressor of no interest. We then extracted the z-scored estimates per time-bin within the previously defined S1hand area ROI. All statistical maps were projected onto a cortical surface using Connectome's Workbench (Marcus et al., 2011).

To test whether multicollinearity between the parameter estimates in our GLM was reasonably low, we calculated the variance inflation factor (VIF). This represents how much the variance of an individual regressor can be explained due to correlation to other regressors in our model (Zuur et al., 2010). For each variable, VIF was computed by the following formula:

$$
\operatorname{VIF}=\frac{\operatorname{Var}(E)}{\operatorname{Var}(X)}
$$

where $\operatorname{Var}(E)$ reflects the mean estimation variance of all regression weights (stimulation and information storage regressors for each finger) while $\operatorname{Var}(X)$ reflects the mean estimation variance in case all regressors would be estimated individually. A VIF of 1 indicates total absence of collinearity between the regressor of interest and all other regressors in our GLM while a large VIF signals a serious collinearity problem. There is no clear threshold for acceptable multicollinearity. Previous literature however recommends that the VIF is ideally smaller than 2.5 (Johnston et al., 2018). In our case, the VIF was on 1.45, averaging across regressors reflecting stimulation one, delay and stimulation two. This indicates that we can disentangle the stimulation and WM delay period activity with appropriate certainty. We additionally computed the VIF for the regressors relating to the stimulation periods and the time-binned delay periods (i.e. stimulation one, delay (0-2s), delay (2-4s), delay (4-6s) and stimulation two). As expected, the resulting VIF was on average 11.38, signaling concerning multicollinearity. Since the WM related activity within the time-bins is correlated, this result was expected, but also means our findings of our time-binned analysis should be interpreted with caution. 
1 We used multivariate pattern analysis (MVPA) to decode which finger was stimulated based

2 on activity during the delay period. This analysis was conducted for voxels within the $\mathrm{S} 1$ hand

3 area mask that have been shown to possess fine-grained finger representations. First-level

4 parameter estimates were computed for all events of each trial and each participant using a

5 voxel-based general linear model (GLM) in SPM (v12) based on the gamma hemodynamic

6 response function. This resulted in 192 beta estimates (48 trials $\times 4$ runs) during the delay

7 period per participant across both conditions. 96 beta estimates for each memory and no 8 memory condition.

We trained a linear classifier (support vector machine, SVM) to predict which finger was stimulated in a specific trail based on the respective delay period activity using the nilearn toolbox (Abraham et al., 2014). We calculated classification accuracies using a leaveone-run-out cross-validation approach. The accuracies were averaged across folds, resulting in one accuracy per condition and per participant. To approximate the true chance level, we shuffled the condition labels using 1000 permutations (Ojala \& Garriga, 2009), as implemented in the scikitlearn toolbox (Pedregosa, 2011). Then, we calculated permutationbased $p$-values based on the following formula:

(2) $(C+1) /\left(n \_\right.$permutations +1$)$,

18 where $C$ is the number of permutation scores that were higher than the true accuracy. To assess the statistical significance of each ROl's decoding accuracy, we used one sample ttests against the approximated chance level (Stelzer et al., 2013). We further used a paired ttests to compare classification accuracies between the WM and non-WM conditions.

Finally, to assess whether somatotopic representations of stimulus content determined participant's behavioral performance, we computed classification accuracies during both stimulation and the delay period for each run and the respective behavioral performance. Then we calculated a repeated-measures correlation coefficient (Bakdash \& Marusich, 2017). For the purpose of exploration, we obtained coefficients for different timepoints, during both stimulations as well as the delay period.

To detect outliers we implemented a state-of-the-art method using robustbase (Finger, 2010). $\mathbf{S}_{\mathbf{n}}$ identifies an outlier ( $\mathrm{x}_{\mathbf{i}}$ ) if the median distance of $\mathrm{x}_{\mathbf{i}}$ from all other points, was greater than the outlier criterion $(\lambda=4)$ times the median absolute distance of every point from every other point: 
(1) $\frac{\operatorname{med}_{j \neq i}\left|x_{i}-x_{j}\right|}{S_{n}}>\lambda$ where $S_{n}=c_{n_{i=1: n}}^{\operatorname{med}}\left\{\begin{array}{c}m e d \\ j \neq 1\end{array} \mid x_{i}-x_{j}\right\}$,

2 where $c_{n}$ is a bias correction factor for finite sample sizes (Rousseeuw \& Croux, 1993). We

3 detected no outliers for behavioral data that had to be excluded from any further analysis.

4 Before conducting any ANOVA testing, we validated the assumptions for homogeneity of 5 variances and normal distributions using a Levene's and Shapiro-Wilk test. Effect sizes of 6 different variables were measured using eta squared. ANOVA analysis was done using the 7 bioinfo-kit and pingouin toolbox (Huang et al., 2014; Vallat, 2018).

When computing t-statistics, in order to correct for multiple comparisons we applied

9 a false discovery rate (FDR) method (Benjamini \& Hochberg, 1995).

\section{RESULTS}

A two-way ANOVA showed that behavioral performances differed significantly between frequency differences $\left(F(2,156)=5.06, p<.001, \eta^{2}=0.06\right)$; Fig. 2A), but not between stimulated fingers $\left(F(1,156)=.44, p=.51, \eta^{2}<0.01\right)$. The frequency effect was not significantly different between stimulated fingers (i.e., no interaction effect; $F(1,156)=$ $.88, p=.42, \eta^{2}=0.01$ ). A post hoc test (Tuckey's HSD) on pairwise comparisons on frequency differences pairs revealed that discrimination accuracy was significantly different between 2 and $6 \mathrm{~Hz}$ differences $(q=4.48, p<.01)$ and showed no significant difference for the rest of the pairs (4 $\mathrm{Hz}$ vs $6 \mathrm{~Hz}: q=2.67, p=.15$ and $2 \mathrm{~Hz}$ vs $4 \mathrm{~Hz} q=1.81, p=.41$ ).

A

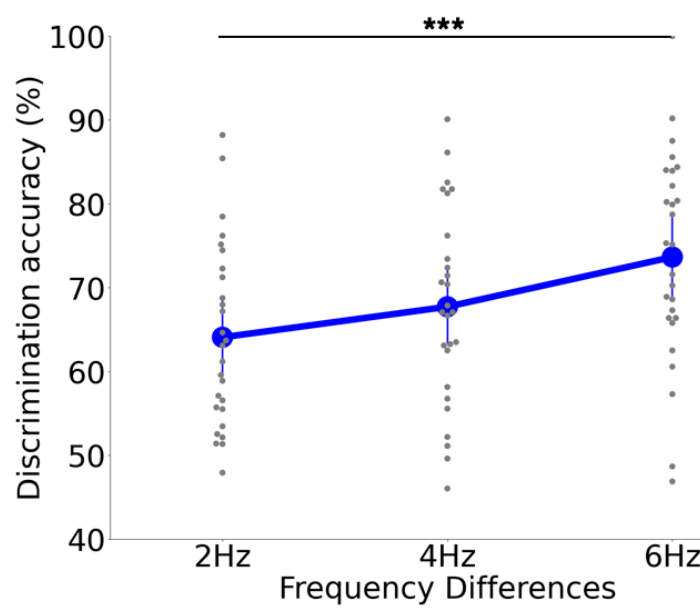

B

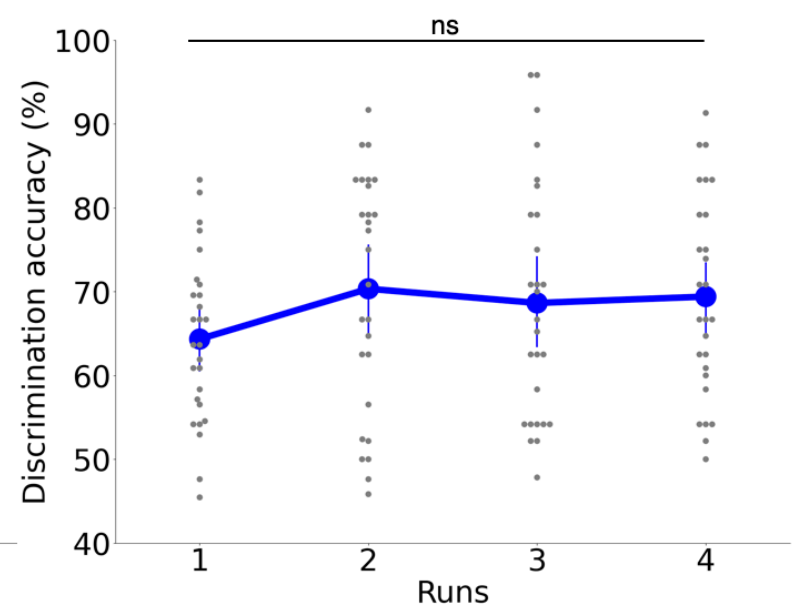


Fig. 2. Behavioral performance results. A. Discrimination accuracy (\% of correct answers) improved when frequency differences were larger. The blue dots reflect the group mean and the blue error bars indicate the standard deviation of the discrimination accuracy per frequency difference across the whole experiment. B. Behavioral performance was not significantly different across runs. The blue dots reflect the group mean and the blue error bars indicate the standard deviation of discrimination accuracies across each run. This demonstrates that our SDT criterion assured stable discrimination accuracies across runs. Grey dots represent individual participants' results. ${ }^{* \star \star}=p<.001$; ns $=$ nonsignificant.

We first determined brain areas that were more activate during the delay period in the memory compared to the no memory condition. We found that WM processing involved a distributed brain network: i.e., bilateral frontal lobe, bilateral (medial/inferior) frontal gyrus (MFG, IFG), bilateral pre-motor cortex and supplementary motor area (PMC, SMA), bilateral inferior parietal lobule (IPL), bilateral superior parietal lobule (SPL), bilateral supramarginal gyrus (SMG), bilateral caudate, bilateral thalamus, bilateral nucleus accumbens, and bilateral insula (Fig. 3A and Table 1). As in previous human fMRI studies, this univariate analysis did not reveal any $\mathrm{S} 1$ activity.

Furthermore, we contrasted activity levels during vibrotactile stimulation between fingers (i.e., index>little and little>index finger), and, as expected, observed separated finger representations in contralateral S1(Fig. 3B, top). More specifically, there was stronger index compared to little finger activity on the lateral part of $\mathbf{S 1}$, while there was stronger little compared to index finger activity medial of S1. These S1 activity maps are in line with previous findings on finger somatotopy which is highly specific within S1 (Besle et al., 2013; Kikkert et al., 2021; Kolasinski et al., 2016; Martuzzi et al., 2014; Sanchez Panchuelo et al., 2018; Sanders et al., 2019). Finally, we assessed whether finger-specific activity was sustained within these finger-specific maps during the delay period. We did not find any significant selective activity in these finger maps during the delay period (index $>$ rest: $t=.6$, $p=.52$, little finger $>$ rest: $t=1.5, p=.14)$ (Fig. 3B, bottom). 
A

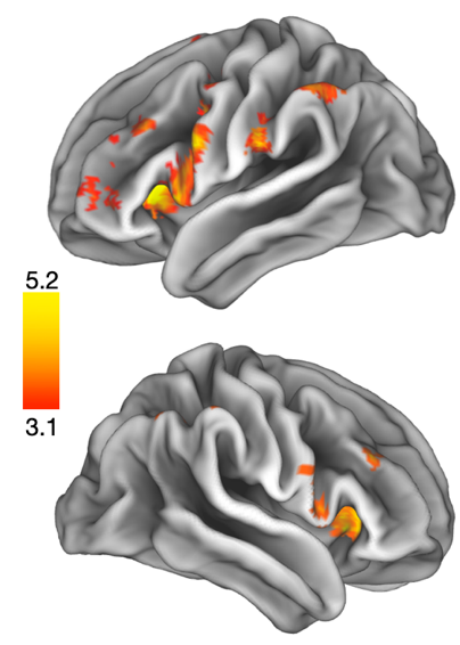

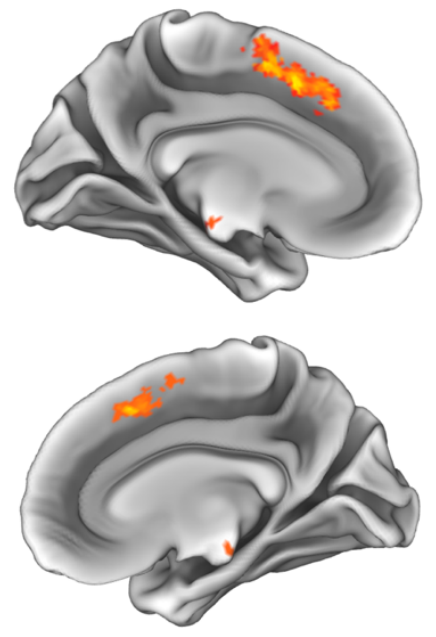

B

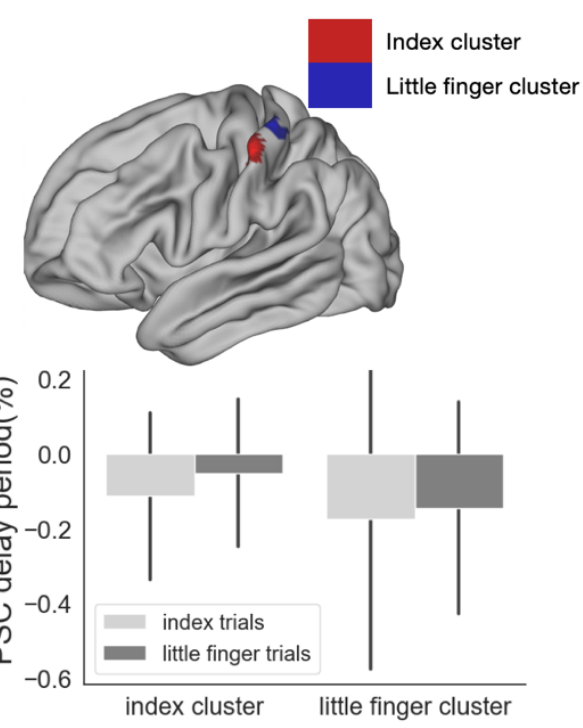

2 Fig. 3. Univariate group results. A. We determined brain regions that were more activate during the 3 delay period of the memory compared to the no memory condition. A statistical map $(Z>3.1)$ was obtained by contrasting delay period activity in memory trials to no memory trials. The map was separately projected onto a cortical surface contralateral (top) and ipsilateral (bottom) to the stimulus site. WM related activity resided in a network of brain regions (for more details, see Table 1). B. S1 areas activated during index (red) and little (blue) finger stimulation (top). Brain regions activated during index and little finger stimulation Z-statistic images were thresholded using clusters determined by Z $>3.1, p<.05$ family-wise-error-corrected (FWE) cluster significance and were projected onto a cortical surface. Finger maps were located in contralateral SI. We then extracted the percent signal change (PSC) within these finger-specific S1 areas during the delay period by contrasting activity during each finger stimulation to rest (bottom). The PSC was only extracted from the pre-defined finger maps in left SI. Bar plots are centered at the mean and error bars reflect the standard deviation.

$\begin{array}{lccll}\text { Left Frontal Pole } & -42 & 52 & 2 & 4.33 \\ \text { Right Frontal Pole } & 40 & 46 & 32 & 3.86 \\ \text { Left IFG } & -52 & 16 & 2 & 5.14 \\ \text { Right IFG } & 58 & 10 & 18 & 4.59 \\ \text { Left MFG } & -44 & 30 & 34 & 4.45 \\ \text { Left PMC (+SMA) } & -4 & 8 & 50 & 5.49\end{array}$




\begin{tabular}{|c|c|c|c|c|c|}
\hline Right PMC (+SMA) & 8 & 20 & 46 & 5.78 & $V$ \\
\hline Left IPS & -42 & -52 & 48 & 5.14 & \\
\hline Left IPL & -56 & -20 & 28 & 4.78 & \\
\hline Right IPL & 46 & -48 & 52 & 4.86 & \\
\hline Left SMG & -42 & -46 & 40 & 4.77 & \\
\hline Right SMG & 51 & -32 & 46 & 4.16 & \\
\hline Left SPL & -50 & -48 & 58 & 4.64 & \\
\hline Left Insular cortex & -30 & 24 & 6 & 5.61 & \\
\hline Right Insular cortex & 36 & 22 & -2 & 5.41 & \\
\hline Left Nucleus Accumbens & -12 & 14 & -6 & 5.27 & \\
\hline Right Nucleus Accumbens & 10 & 12 & -4 & 4.16 & \\
\hline Left Putamen & -18 & 6 & -10 & 4.96 & \\
\hline Right Putamen & 20 & 10 & -8 & 4.34 & \\
\hline Left Caudate & -12 & 18 & 0 & 4.80 & \\
\hline Right Caudate & 14 & 18 & 4 & 4.97 & \\
\hline Left Thalamus & -8 & -24 & -16 & 3.86 & \\
\hline Right Thalamus & 2 & -20 & -12 & 3.91 & \\
\hline
\end{tabular}

Table 1. Identified brain regions in which the local activity during the delay period reflected tactile WM processing. Sorted from anterior to posterior and from cortical to subcortical. A statistical map was obtained by contrasting BOLD responses in memory trials to no memory trials during the delay period. In a next step we parametrically modulated the delay activity, assuming a U-shaped activity during the delay period. Brain regions overlapping with the initial results are indicated by a check mark in the right column. All z-statistic images were thresholded using clusters determined by $Z>3.1$ and $p<.05$ family-wise-error-corrected (FWE) cluster significance. Mean Fischer Z indicates peak z-values. Areas were labeled according to the Juelich Histological Atlas and Havard-Oxford (Sub-)cortcial Structural Atlas (Eickhoff et al., 2005). MFG= medial frontal gyrus IFG = inferior frontal gyrus, PMC = pre-motor cortex, SMA = supplementary motor area, IPL = inferior parietal lobule, $\mathrm{SPL}=$ superior parietal lobule, SMG = supramarginal gyrus.

We then examined how brain activity temporally changed during the delay period. We parametrically modulated the delay period regressors by the hypothesized U-shaped activity changes and computed the associated statistical maps. A U-shaped activity modulation was found in a similar network of brain regions as in Fig. 4A. Interestingly, this modulation also elicited activity maps in S1 and secondary somatosensory cortex (SII). For visualization 
1 purposes only, we extracted the activity at each time bin (i.e., from a separate GLM not 2 involving a parametric modulation regressor) within the contralateral $\mathrm{S} 1$ hand area to 3 demonstrate U-shaped activity across time bins (Fig. 4B).

A

4

5
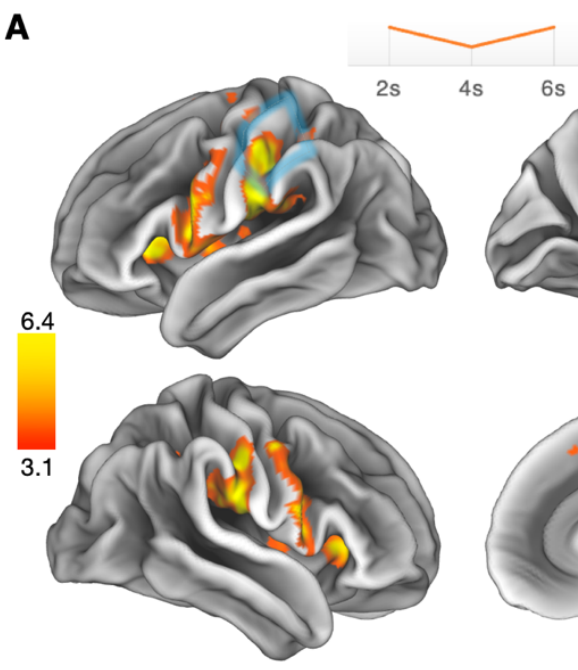

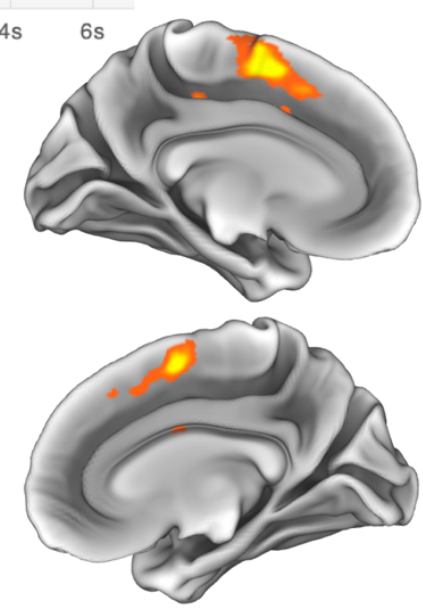

B

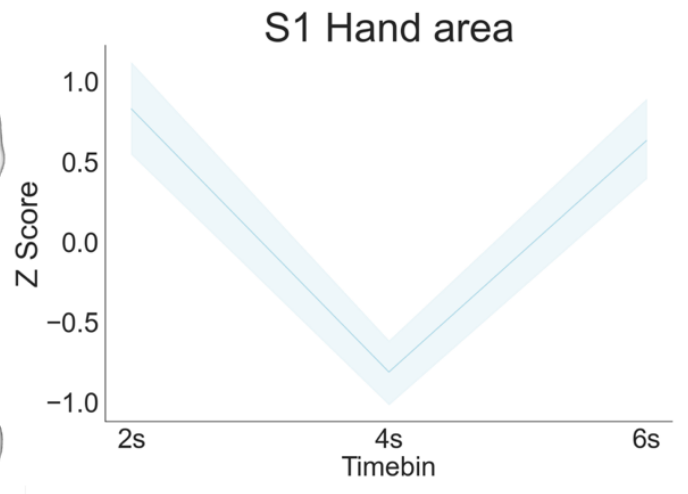

Fig. 4. U-shaped parametric modulation of brain activity during the delay period. A Brain regions exhibiting u-shaped modulated delay activity patterns during the delay period (2-6s) (see insert at the top reflecting the parametric modulator entered into the GLM). The area highlighted in blue represents the S1 hand area. B. For visualization purposes only, each time point between 2 and 6 s of the delay period (each time bin corresponding to one TR) was modeled as a separate regressor in an univariate GLM. Z scores of the beta estimates were extracted from the $\mathrm{S} 1$ hand area for each time bin of the delay period and averaged across participants. Blue shading reflects the $95 \%$ confidence interval.

\section{FINGER-SPECIFIC S1 WM REPRESENTATIONS}

We hypothesized that executing a WM task would modulate finger specific representations in $\mathrm{S1}$. While univariate analysis may not allow us to find WM-related activity in S1 during the delay period, MVPA is more sensitive to detecting subtle differences in activity patterns (Weaverdyck et al., 2020), making it an interesting approach to further examine our hypotheses. We decoded the stimulated finger (e., index versus little finger) during stimulation 1(f1), during the delay period, and during stimulation 2 (f2) separately for memory and no memory trials (Fig. 5). We did this in contralateral S1, which has shown to possess fine-grained finger representations (Besle et al., 2013; Cunningham et al., 2013; Martuzzi et al., 2014; Sanchez Panchuelo et al., 2018; Sanders et al., 2019), and a control white matter ROI.

During the both stimulations ( $\mathrm{f} 1$ and f2) of memory trials, classification accuracies in contralateral $\mathrm{S} 1$ hand area were significantly higher than chance level (f1: $t=14.25, p<.001$, 
1 f2: $t=13.79, p<.001$ ) which was also the case for during no memory trials ( $f 1: t=11.81, p$ $<.001, \mathrm{f} 2: t=10.76, p<.001)$.

Classification accuracies differed significantly between timepoints (two-way ANOVA; $\left.F(2,156)=103.7, p<.001, \eta^{2}=.57\right)$, and conditions $\left(F(1,156)=12.14, p<.01, \eta^{2}=.07\right)$. The time effect was not significantly different between conditions (i.e., no interaction; $F(2$, $\left.156)=.11, p=.9, \eta^{2}<0.01\right)$. More specifically, during both stimulations $(\mathrm{f} 1, \mathrm{f} 2)$ classification accuracies inferred from the $\mathrm{S} 1$ hand area were significantly higher during memory trials compared to no memory trials (f1: $t=4.28, p<.001$; $\mathrm{f} 2: t=4.07, p<.001$ ).

Classification accuracies derived from the control ROI (White matter mask) did not show any significant differences between timepoints $\left(F(2,156)=1.84, p=.16, \eta^{2}=.02\right)$ nor conditions $\left(F(1,156)=2.11, p=p=.15, \eta^{2}=.01\right)$, however exhibited an interaction effect $\left(\mathrm{F}(2,156)=4.8, \mathrm{p}=\mathrm{p}<.05, \eta^{2}=.06\right)$.

During the WM delay period classification accuracy in the $S 1$ hand area was significantly higher than chance level $(t=4.55, p<.001)$ which was not the case for the no memory delay period ( $t=1.54, p=.14$ ). Moreover, classification accuracies significantly varied between memory conditions in the $S 1$ hand area $(t=2.91, p<.01)$. This was not the case in our control ROI where classification accuracies did not differ from chance level for both conditions (memory: $t=.04, p=.97$; no memory: $t=-.36, p=.72$ ) nor between the memory and no memory conditions $(t=.02, p=.98)$. These results suggest that $\mathrm{S} 1$, which possess especially fine-grained finger somatotopy, stores information about the stimulus site during WM delay and in the absence of any tactile input.

A

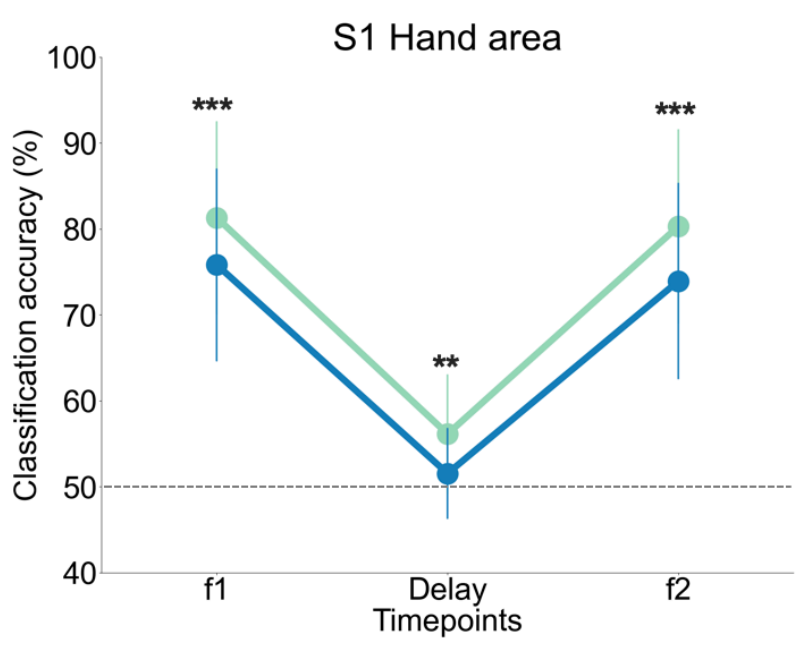

B

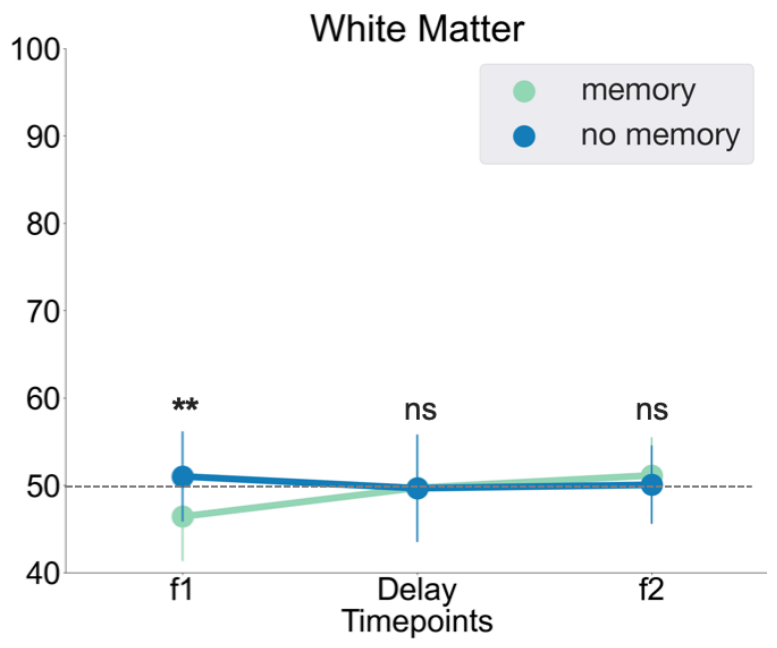

Fig. 5. MVPA results on somatotopic modulations. We extracted activity patterns to predict what 
permutations where we shuffled the finger labels. We ran these permutations separately for each region of interest and condition (memory vs. no memory). A. Classification accuracies based on activity patterns in hand area within contralateral primary somatosensory cortex. B. Classification accuracies based on activity patterns within white matter as control ROI, where no somatotopy is assumed. The point plots are centered at the mean and error bars reflect the standard deviation. Grey dotted lines reflect the theoretical chance level. T-statistics results between conditions (memory vs. no memory) are indicated by ${ }^{*} \mathrm{p}<.05,{ }^{* *} \mathrm{p}<.01{ }^{* * *} \mathrm{p}<.001$.

\section{DISCUSSION}

In the present study, we investigated S1 activity during a vibrotactile WM task through the lens of somatotopic representations. More specifically, we explored whether finger specific information in $\mathrm{S} 1$ is modulated by cognitive processes relevant for performing a WM vibrotactile discrimination task. Even though BOLD activity in $\mathrm{S} 1$ did not differ from rest, we could show more specific representational tuning when participants had to retain taskrelevant information. Using MVPA we were able to decode for which finger information was kept in WM during the delay period, demonstrating that finger-specific activity patterns resided in the contralateral S1 hand area in the absence of any tactile stimulation. Importantly, finger-specific classification accuracies during the delay period were not merely driven by residue activity from the preceding tactile stimulation. Indeed, classification accuracies were not higher than chance in a no memory condition in which participants received identical stimulation as in the memory condition, but were not required to keep any tactile information in WM. Lower classification accuracies during no memory trials indicates that S1 activity was less finger specific suggesting that tuning curves were broader.

\section{SOMATOTOPIC MODULATION IN S1 IS MAINTAINED DURING WM DELAY PERIOD}

This modulation of finger representation in contralateral S1 appears to also occur during WM. Pasternak and Greenlee (2005) analyzed a wide range of non-human primate studies and found that sensory WM recruits a wide circuitry of brain areas including sensory brain regions, which is in line with our observations. Neurophysiological research in non-human primates have shown that $\mathrm{S} 1$ cells are not only involved in perception of tactile stimuli, but also sustain

30 their firing during the WM delay period (Katus et al., 2015; Y. D. Zhou \& Fuster, 1996; Y.-D. 31 Zhou \& Fuster, 2000). This suggests that sensory brain regions not only process sensory stimuli, but also retain and manipulate sensory information in the absence of any sensory input, also known as 'sensory recruitment' models. According to these models, vibrotactile 
1 information is processed in $\mathrm{S} 1$, relayed to $\mathrm{S} 2$, which possesses a reciprocal connection with

$2 \quad \mathrm{~S} 1$ and disseminates information via insular projections to frontal and parietal regions (Wang

3 et al., 2013). This account is in line with our parametric mapping results. We found a whole

4 network to be active during WM retrieval including somatosensory and fronto-partial, insular 5 cortices as well as subcortical regions.

Furthermore, previous findings indicate that tactile WM operates within somatotopically organized sensory brain regions (Harris et al., 2001; Katus et al., 2015; Schmidt \& Blankenburg, 2018). However, till date, it has remained unknow whether WM modulates S1 in a somatotopic fashion. Our results indicate that finger-specific information is retained during the delay period despite the absence of any tactile input, suggesting WM content representation in contralateral S1. No such finger-specific information content was observed during the delay period in the no memory control condition.

It is uncertain which specific cognitive mechanism might drive our result. Indeed, does finger-specific representational tuning during the delay period reflect the maintenance of the stimulus features in WM or is it driven by other cognitive processes? Attention is an integral part of performing WM tasks (Cowan et al., 2013; Logie \& Cowan, 2015; Schmidt \& Blankenburg, 2018, 2019). By directing attention, the brain controls which retained information is in a state of availability and one important aspect of WM is to make information temporally accessible to cognitive control instead of merely acting as an information buffer system. With regard to representational tuning in $\mathrm{S} 1$, it has been shown that when a spot on the body surface (e.g. fingertips) was being attended to, preparatory tuning of corresponding area in S1 was observed (Nelson et al., 2004; Puckett et al., 2017; Roland, 1981; Sterr et al., 2007). Similarly in our experiment, when participants were instructed to ignore vibrotactile stimuli during no memory trials, no representational tuning was observed even though the tactile stimuli were identical. Our findings further highlight that activity in S1 and S2 contralateral to the stimulated finger exhibits a U-shaped activity profile across the delay period, which might provide a glimpse into the temporal modulation of a putative attentional mechanism. Interestingly, S1 and S2 were not the only areas that exhibited a U-shaped profile. BOLD responses in fronto-parietal and subcortical regions (i.e. caudate and thalamus) also appeared to reflect this U-shaped activity profile. Task-relevant modulation could occur through prefrontal cortex (PFC) by gating the flow of vibrotactile information to S1. Indeed, the prefrontal-thalamic inhibitory system could play a key role in boosting relevant stimulus information while reducing irrelevant ones (Staines et al., 2002). It is likely that the final increase in delay activity might be driven by anticipation of increasing attentional demands, although we jittered the length of the delay period to prevent for anticipatory activity (Rose et 
1 al., 2016). Therefore, we cannot rule out that attentional mechanisms are driving our results.

2 As mentioned before, WM and attention are thought to be closely intertwined (Cowan et al.,

3 2013; Logie \& Cowan, 2015) and fMRI studies might not be the appropriate approach to test

4 these subtle differences on a synaptic level (Stokes, 2015).

Our results suggest that our $\mathrm{S} 1$ decoding results reflect representational tuning that did not merely arise from carry-over effects caused by stimulus encoding. Our memory delay period was relatively short (6-8s) compared to other $\mathrm{fMRI}$ studies investigating vibrotactile WM that often employ delays of 12 s to avoid any carry-over effects (Schmidt \& Blankenburg, 2018; Wu et al., 2018). However, it is unlikely that the WM related somatotopic activity patterns observed in S1 were merely a residue of stimulus evoked activity due to the sluggishness of the BOLD response. If we merely decoded a residue of the tactile stimulation per se, then we would expect similar decoding results for memory and no memory conditions during the delay period. Indeed, finger stimulations were identical for the memory and no memory conditions. The only difference between these conditions was the cognitive strategy participants employed. While decoding accuracy was higher than chance in the delay period for the memory condition, this was not the case for the no memory condition. Furthermore, we found a significant difference in decoding accuracies between conditions. Moreover, the low VIF of the regressors suggested that activity related to stimulus perception and WM storage could be disentangled by our model.

Together our results show that $S 1$ plays a role in vibrotactile frequency discrimination. Recently, two transcranial magnetic stimulation (TMS) studies found that behavioral vibrotactile frequency discrimination performance was impaired if TMS pulses were applied to either contralateral S1 during the delay period (Harris et al., 2002). This hints at the idea that the involvement of $\mathrm{S} 1$ and modulation of its somatotopic maps might be crucial to discriminate between tactile stimuli perceived on different fingers.

\section{CONCLUSION}

Tactile decision making based on mentally represented information is an essential part of higher cognitive processes. In the absence of sensory stimuli, decisions are made with information stored in WM (Christophel et al., 2017). A more profound insight into how the brain stores information in WM is essential to understand human cognition. This includes not 
only the involvement of sensory regions in WM but also how their topographic organization might be modulated for information storage. Together, our results show that vibrotactile WM (i) recruits a distributed network of brain regions, (ii) modulates delay activity in various brain regions and (iii) modulates somatotopic finger representations in contralateral $\mathrm{S} 1$. These insights aid our understanding on how cognitive processes involved in WM shape cortical sensory activation with potential implications for individuals with WM impairments.

\section{ACHKNOLEDGMENTS}

We thank Alain Plüss and Bianca Badii for help with data collection. This work was supported by the Swiss National Science Foundation (320030_175616) and by the National Research Foundation, Prime Minister's Office, Singapore under its Campus for Research Excellence and Technological Enterprise (CREATE) program (FHT). SK was supported by the ETH Zürich

Postdoctoral Fellowship Program.

\section{REFERENCES}

Abraham, A., Pedregosa, F., Eickenberg, M., Gervais, P., Mueller, A., Kossaifi, J., Gramfort, A., Thirion, B., \& Varoquaux, G. (2014). Machine learning for neuroimaging with scikit-learn. Frontiers in Neuroinformatics, 8. https://doi.org/10.3389/fninf.2014.00014

Ariani, P., Pruszynski, J. A., \& Diedrichsen, J. (2021). Motor planning brings human primary somatosensory cortex into action-specific preparatory states. BioRxiv.

Bakdash, J. Z., \& Marusich, L. R. (2017). Repeated Measures Correlation. Frontiers in Psychology, 8. https://doi.org/10.3389/fpsyg.2017.00456

Benjamini, Y., \& Hochberg, Y. (1995). Controlling the False Discovery Rate: A Practical and Powerful Approach to Multiple Testing. Journal of the Royal Statistical Society: Series B (Methodological), 57(1). https://doi.org/10.1111/j.2517-6161.1995.tb02031.x

Besle, J., Sánchez-Panchuelo, R.-M., Bowtell, R., Francis, S., \& Schluppeck, D. (2013). Single-subject fMRI mapping at $7 \mathrm{~T}$ of the representation of fingertips in S1: a comparison of event-related and phase-encoding designs. Journal of Neurophysiology, 109(9). https://doi.org/10.1152/jn.00499.2012

Braver, T. S., Cohen, J. D., Nystrom, L. E., Jonides, J., Smith, E. E., \& Noll, D. C. (1997). A Parametric Study of Prefrontal Cortex Involvement in Human Working Memory. NeuroImage, 5(1). https://doi.org/10.1006/nimg.1996.0247

Christophel, T. B., Klink, P. C., Spitzer, B., Roelfsema, P. R., \& Haynes, J.-D. (2017). The Distributed Nature of Working Memory. Trends in Cognitive Sciences, 21(2). https://doi.org/10.1016/j.tics.2016.12.007

Cowan, N., Blume, C. L., \& Saults, J. S. (2013). Attention to attributes and objects in working memory. Journal of Experimental Psychology: Learning, Memory, and Cognition, 39(3). https://doi.org/10.1037/a0029687

Cunningham, D. A., Machado, A., Yue, G. H., Carey, J. R., \& Plow, E. B. (2013). Functional somatotopy revealed across multiple cortical regions using a model of 
complex motor task. Brain Research, 1531. https://doi.org/10.1016/j.brainres.2013.07.050

D'Esposito, M., \& Postle, B. R. (2015). The Cognitive Neuroscience of Working Memory. Annual Review of Psychology, 66(1). https://doi.org/10.1146/annurevpsych-010814-015031

Detorakis, G. Is., \& Rougier, N. P. (2014). Structure of receptive fields in a computational model of area $3 \mathrm{~b}$ of primary sensory cortex. Frontiers in Computational Neuroscience, 8. https://doi.org/10.3389/fncom.2014.00076

Diedrichsen, J., Wiestler, T., \& Ejaz, N. (2013). A multivariate method to determine the dimensionality of neural representation from population activity. NeuroImage, 76. https://doi.org/10.1016/j.neuroimage.2013.02.062

Eickhoff, S. B., Stephan, K. E., Mohlberg, H., Grefkes, C., Fink, G. R., Amunts, K., \& Zilles, K. (2005). A new SPM toolbox for combining probabilistic cytoarchitectonic maps and functional imaging data. NeuroImage, 25(4). https://doi.org/10.1016/j.neuroimage.2004.12.034

Ejaz, N., Hamada, M., \& Diedrichsen, J. (2015). Hand use predicts the structure of representations in sensorimotor cortex. Nature Neuroscience, 18(7). https://doi.org/10.1038/nn.4038

Ester, E. F., Serences, J. T., \& Awh, E. (2009). Spatially Global Representations in Human Primary Visual Cortex during Working Memory Maintenance. Journal of Neuroscience, 29(48). https://doi.org/10.1523/JNEUROSCI.4388-09.2009

Finger, R. (2010). Review of 'Robustbase' software for R. Journal of Applied Econometrics, 25(7). https://doi.org/10.1002/jae.1194

Fischl, B. (2012). FreeSurfer. NeuroImage, 62(2). https://doi.org/10.1016/j.neuroimage.2012.01.021

Fischl, B., Sereno, M. I., \& Dale, A. M. (1999). Cortical Surface-Based Analysis. NeuroImage, 9(2). https://doi.org/10.1006/nimg.1998.0396

Greve, D. N., \& Fischl, B. (2009). Accurate and robust brain image alignment using boundary-based registration. NeuroImage, 48(1), 63-72. https://doi.org/10.1016/j.neuroimage.2009.06.060

Guan, C., Aflalo, T., Zhang, C. Y., Rosario, E. R., Pouratian, N., \& Andersen, R. A. (2021). Preserved motor representations after paralysis. BioRxiv.

Harris, J. A., Arabzadeh, E., Fairhall, A. L., Benito, C., \& Diamond, M. E. (2006). Factors Affecting Frequency Discrimination of Vibrotactile Stimuli: Implications for Cortical Encoding. PLoS ONE, 1(1). https://doi.org/10.1371/journal.pone.0000100

Harris, J. A., Harris, I. M., \& Diamond, M. E. (2001). The Topography of Tactile Working Memory. The Journal of Neuroscience, 21(20). https://doi.org/10.1523/JNEUROSCI.21-20-08262.2001

Harris, J. A., Miniussi, C., Harris, I. M., \& Diamond, M. E. (2002). Transient Storage of a Tactile Memory Trace in Primary Somatosensory Cortex. The Journal of Neuroscience, 22(19). https://doi.org/10.1523/JNEUROSCI.22-19-08720.2002

Huang, Y., Wang, J. Y., Wei, X. M., \& Hu, B. (2014). Bioinfo-Kit: A Sharing Software Tool for Bioinformatics. Applied Mechanics and Materials, 472. https://doi.org/10.4028/www.scientific.net/AMM.472.466

Jenkinson, M. (2002). Improved Optimization for the Robust and Accurate Linear Registration and Motion Correction of Brain Images. NeuroImage, 17(2). https://doi.org/10.1016/S1053-8119(02)91132-8

Jenkinson, M., \& Smith, S. M. (2001). A global optimization method for robust affine registration of brain images. Medical Imaging Analysis, 5, 143-156. 
Johnston, R., Jones, K., \& Manley, D. (2018). Confounding and collinearity in regression analysis: a cautionary tale and an alternative procedure, illustrated by studies of British voting behaviour. Quality \& Quantity, 52(4). https://doi.org/10.1007/s11135-017-0584-6

Kaas, J. H. (1993). The functional organization of somatosensory cortex in primates. Annals of Anatomy - Anatomischer Anzeiger, 175(6). https://doi.org/10.1016/S0940-9602(11)80212-8

Kaas, J. H. (1997). Topographic Maps are Fundamental to Sensory Processing. Brain Research Bulletin, 44(2). https://doi.org/10.1016/S0361-9230(97)00094-4

Katus, T., Grubert, A., \& Eimer, M. (2015). Electrophysiological Evidence for a Sensory Recruitment Model of Somatosensory Working Memory. Cerebral Cortex, 25(12). https://doi.org/10.1093/cercor/bhu153

Kikkert, S., Pfyffer, D., Verling, M., Freund, P., \& Wenderoth, N. (2021). Finger somatotopy is preserved after tetraplegia but deteriorates over time. ELife, 10. https://doi.org/10.7554/eLife.67713

Kolasinski, J., Makin, T. R., Jbabdi, S., Clare, S., Stagg, C. J., \& Johansen-Berg, H. (2016). Investigating the Stability of Fine-Grain Digit Somatotopy in Individual Human Participants. Journal of Neuroscience, 36(4). https://doi.org/10.1523/JNEUROSCI.1742-15.2016

Kuehn, E., Haggard, P., Villringer, A., Pleger, B., \& Sereno, M. I. (2018). VisuallyDriven Maps in Area 3b. The Journal of Neuroscience, 38(5). https://doi.org/10.1523/JNEUROSCI.0491-17.2017

Logie, R. H., \& Cowan, N. (2015). Perspectives on working memory: introduction to the special issue. Memory \& Cognition, 43(3). https://doi.org/10.3758/s13421-0150510-x

Marcus, D. S., Harwell, J., Olsen, T., Hodge, M., Glasser, M. F., Prior, F., Jenkinson, M., Laumann, T., Curtiss, S. W., \& van Essen, D. C. (2011). Informatics and Data Mining Tools and Strategies for the Human Connectome Project. Frontiers in Neuroinformatics, 5. https://doi.org/10.3389/fninf.2011.00004

Martuzzi, R., van der Zwaag, W., Farthouat, J., Gruetter, R., \& Blanke, O. (2014). Human finger somatotopy in areas $3 \mathrm{~b}, 1$, and 2: A $7 \mathrm{~T}$ fMRI study using a natural stimulus. Human Brain Mapping, 35(1). https://doi.org/10.1002/hbm.22172

Mountcastle, V. B., Talbot, W. H., Darian-Smith, I., \& Kornhuber, H. H. (1967). Neural Basis of the Sense of Flutter-Vibration. Science, 155(3762). https://doi.org/10.1126/science.155.3762.597

Nelson, A. J., Staines, W. R., Graham, S. J., \& McIlroy, W. E. (2004). Activation in SI and SII; the influence of vibrotactile amplitude during passive and task-relevant stimulation. Cognitive Brain Research, 19(2). https://doi.org/10.1016/j.cogbrainres.2003.11.013

Ojala, M., \& Garriga, G. C. (2009, December). Permutation Tests for Studying Classifier Performance. 2009 Ninth IEEE International Conference on Data Mining. https://doi.org/10.1109/ICDM.2009.108

Pasternak, T., \& Greenlee, M. W. (2005). Working memory in primate sensory systems. Nature Reviews Neuroscience, 6(2). https://doi.org/10.1038/nrn1603

Pedregosa, F. and V. G. and G. A. and M. V. and T. B. and G. O. and B. M. and P. P. and W. R. and D. V. and V. J. and P. A. and C. D. and B. M. and P. M. and D. E. (2011). Scikit-learn: Machine Learning in Python. Journal of Machine Learning Research, 12(85), 2825-2830.

Peirce, J., Gray, J. R., Simpson, S., MacAskill, M., Höchenberger, R., Sogo, H., Kastman, E., \& Lindeløv, J. K. (2019). PsychoPy2: Experiments in behavior made 
easy. Behavior Research Methods, 51(1). https://doi.org/10.3758/s13428-01801193-y

PENFIELD, W., \& BOLDREY, E. (1937). SOMATIC MOTOR AND SENSORY REPRESENTATION IN THE CEREBRAL CORTEX OF MAN AS STUDIED BY ELECTRICAL STIMULATION. Brain, 60(4). https://doi.org/10.1093/brain/60.4.389

Preuschhof, C., Heekeren, H. R., Taskin, B., Schubert, T., \& Villringer, A. (2006). Neural Correlates of Vibrotactile Working Memory in the Human Brain. Journal of Neuroscience, 26(51). https://doi.org/10.1523/JNEUROSCI.2767-06.2006

Puckett, A. M., Bollmann, S., Barth, M., \& Cunnington, R. (2017). Measuring the effects of attention to individual fingertips in somatosensory cortex using ultra-high field (7T) fMRI. NeuroImage, 161. https://doi.org/10.1016/j.neuroimage.2017.08.014

Roland, P. E. (1981). Somatotopical tuning of postcentral gyrus during focal attention in man. A regional cerebral blood flow study. Journal of Neurophysiology, 46(4). https://doi.org/10.1152/jn.1981.46.4.744

Rose, N. S., LaRocque, J. J., Riggall, A. C., Gosseries, O., Starrett, M. J., Meyering, E. E., \& Postle, B. R. (2016). Reactivation of latent working memories with transcranial magnetic stimulation. Science, 354(6316). https://doi.org/10.1126/science.aah7011

Rousseeuw, P. J., \& Croux, C. (1993). Alternatives to the Median Absolute Deviation. Journal of the American Statistical Association, 88(424). https://doi.org/10.1080/01621459.1993.10476408

Sanchez Panchuelo, R. M., Besle, J., Schluppeck, D., Humberstone, M., \& Francis, S. (2018). Somatotopy in the Human Somatosensory System. Frontiers in Human Neuroscience, 12. https://doi.org/10.3389/fnhum.2018.00235

Sanders, Z.-B., Wesselink, D. B., Dempsey-Jones, H., \& Makin, T. R. (2019). Similar somatotopy for active and passive digit representation in primary somatosensory cortex. BioRxiv.

Schmidt, T. T., \& Blankenburg, F. (2018). Brain regions that retain the spatial layout of tactile stimuli during working memory - A 'tactospatial sketchpad'? NeuroImage, 178. https://doi.org/10.1016/j.neuroimage.2018.05.076

Schmidt, T. T., \& Blankenburg, F. (2019). The Somatotopy of Mental Tactile Imagery. Frontiers in Human Neuroscience, 13. https://doi.org/10.3389/fnhum.2019.00010

Schmidt, T. T., Wu, Y., \& Blankenburg, F. (2017). Content-Specific Codes of Parametric Vibrotactile Working Memory in Humans. The Journal of Neuroscience, 37(40). https://doi.org/10.1523/JNEUROSCI.1167-17.2017

Silver, M. A., \& Kastner, S. (2009). Topographic maps in human frontal and parietal cortex. Trends in Cognitive Sciences, 13(11). https://doi.org/10.1016/j.tics.2009.08.005

Smith, S. M. (2002). Fast robust automated brain extraction. Human Brain Mapping, 17(3). https://doi.org/10.1002/hbm.10062

Staines, W. R., Graham, S. J., Black, S. E., \& McIlroy, W. E. (2002). Task-Relevant Modulation of Contralateral and Ipsilateral Primary Somatosensory Cortex and the Role of a Prefrontal-Cortical Sensory Gating System. NeuroImage, 15(1). https://doi.org/10.1006/nimg.2001.0953

Stelzer, J., Chen, Y., \& Turner, R. (2013). Statistical inference and multiple testing correction in classification-based multi-voxel pattern analysis (MVPA): Random permutations and cluster size control. Neurolmage, 65 . https://doi.org/10.1016/j.neuroimage.2012.09.063 
Sterr, A., Shen, S., Zaman, A., Roberts, N., \& Szameitat, A. (2007). Activation of SI is modulated by attention: a random effects fMRI study using mechanical stimuli. NeuroReport, 18(6). https://doi.org/10.1097/WNR.0b013e3280b07c34

Stokes, M. G. (2015). 'Activity-silent' working memory in prefrontal cortex: a dynamic coding framework. Trends in Cognitive Sciences, 19(7). https://doi.org/10.1016/j.tics.2015.05.004

Vallat, R. (2018). Pingouin: statistics in Python. Journal of Open Source Software, 3(31). https://doi.org/10.21105/joss.01026

Wang, L., Bodner, M., \& Zhou, Y.-D. (2013). Distributed neural networks of tactile working memory. Journal of Physiology-Paris, 107(6). https://doi.org/10.1016/j.jphysparis.2013.06.001

Watson, A. B., \& Pelli, D. G. (1983). Quest: A Bayesian adaptive psychometric method. Perception \& Psychophysics, 33(2). https://doi.org/10.3758/BF03202828

Weaverdyck, M. E., Lieberman, M. D., \& Parkinson, C. (2020). Tools of the Trade Multivoxel pattern analysis in fMRI: a practical introduction for social and affective neuroscientists. Social Cognitive and Affective Neuroscience, 15(4). https://doi.org/10.1093/scan/nsaa057

Wiestler, T., \& Diedrichsen, J. (2013). Skill learning strengthens cortical representations of motor sequences. ELife, 2. https://doi.org/10.7554/eLife.00801

Wu, Y., Uluç, I., Schmidt, T. T., Tertel, K., Kirilina, E., \& Blankenburg, F. (2018). Overlapping frontoparietal networks for tactile and visual parametric working memory representations. NeuroImage, 166. https://doi.org/10.1016/j.neuroimage.2017.10.059

Yousry, T. A., Schmid, U. D., Alkadhi, H., Schmidt, D., Peraud, A., Buettner, A., \& Winkler, P. (1997). Localization of the motor hand area to a knob on the precentral gyrus. A new landmark. Brain, 120(1), 141-157. https://doi.org/10.1093/brain/120.1.141

Zhou, Y. D., \& Fuster, J. M. (1996). Mnemonic neuronal activity in somatosensory cortex. Proceedings of the National Academy of Sciences, 93(19). https://doi.org/10.1073/pnas.93.19.10533

Zhou, Y.-D., \& Fuster, J. M. (2000). Visuo-tactile cross-modal associations in cortical somatosensory cells. Proceedings of the National Academy of Sciences, 97(17). https://doi.org/10.1073/pnas.97.17.9777

Zuur, A. F., Ieno, E. N., \& Elphick, C. S. (2010). A protocol for data exploration to avoid common statistical problems. Methods in Ecology and Evolution, 1(1). https://doi.org/10.1111/j.2041-210X.2009.00001.x 